\title{
Updating Predictive Accident Models of Modern Rural Single Carriageway A-roads
}

\author{
Alan Wood \\ School of Engineering, University of Liverpool, $U K$ \\ Linda Mountain
}

School of Engineering, University of Liverpool, UK

Richard D. Connors

Institute for Transport Studies, University of Leeds, UK

Mike Maher

Institute for Transport Studies, University of Leeds, UK

Alan Wood, School of Engineering, University of Liverpool, Brownlow Hill, Liverpool, UK. L69 3GH. alanwood@liverpool.ac.uk 


\title{
Updating Predictive Accident Models of Modern Rural Single Carriageway A-roads
}

\author{
Reliable predictive accident models (PAMs) are essential to design and maintain \\ safe road networks and yet the models most commonly used in the UK were \\ derived using data collected 20 to 30 years ago. Given that the national personal \\ injury accident total fell by some $30 \%$ in the last 25 years, while road traffic \\ increased by over $60 \%$, significant errors in scheme appraisal and evaluation \\ based on the models currently in use seem inevitable. In this paper the temporal \\ transferability of PAMs for modern rural single carriageway A-roads is \\ investigated and their predictive performance is evaluated against a recent data \\ set. Despite the age of these models, the PAMs for predicting the total accidents \\ provide a remarkably good fit to recent data and these are more accurate than \\ models where accidents are disaggregated by type. The performance of the \\ models can be improved by calibrating them against recent data.
}

Keywords: safety, highways, predictive accident model

\section{Introduction}

\subsection{Background}

Reliable predictive accident models (PAMs) are essential to provide and maintain safe road networks. Designers can, for example, use the PAMs incorporated in packages such as ARCADY, OSCADY and PICADY in the appraisal of the safety impacts of alternative design decisions. Planners use COBA and QUADRO in scheme appraisal to estimate the costs of accidents resulting from alternative transport proposals (DfT, 2009), with PAMs essential to forecast accidents with and without possible interventions. Genuine high risk locations can be identified by comparing observed accidents with those predicted by PAMs given the type of site and level of traffic flow. Whereas scheme appraisal takes place prior to implementation using predicted outcomes, evaluation takes place after the event, normally using observed data. In the 
evaluation of safety impacts, however, simple comparisons of observed before and after accidents are known to exaggerate scheme effectiveness because of the regression-tothe-mean effect. This problem can be overcome using an empirical Bayes (EB) approach but its use relies on the availability of suitable PAMs (Mountain et al., 2005; Persaud and Lyon, 2007; Elvik, 2008). However, while the importance of PAMs is clear, the quality of available models is rather less certain.

PAMs are derived by fitting statistical models to data obtained from a large number of road sections or junctions. Such models relate accident frequencies to some measure of exposure (traffic flow) and other observable characteristics that explain the systematic, between-site variation in the number of accidents. Model fitting is by no means straightforward. There is no accepted theory to indicate how accident frequency should increase with traffic flow or, indeed, with other characteristics such as the frequency of accesses along a link; several mathematical functions could be chosen to fit the data (Hauer, 2004). In addition, while the assumption of a negative binomial error structure is now common in accident modelling, this is primarily for mathematical convenience. Recent research suggests that alternative forms of error structure are now not only feasible but may also be more appropriate (Maher and Mountain, 2009). Perhaps the most serious difficulty arises, however, because, over time, there will inevitably be changes in road, vehicle and driver characteristics such that the relationship between the dependent and independent variables will also tend to change. The temporal transferability of PAMs is thus questionable, particularly when the elapsed time is large.

\subsection{Previous studies}

Unfortunately, because the fitting of sophisticated PAMs including a wide range of explanatory variables is not a trivial task, available models tend to be based on data 
collected many years ago. In the case of UK roads, for example, the Transport Research Laboratory (TRL) carried out a comprehensive series of accident studies between the 1970s and 1990s for the UK Department for Transport (DfT), developing a suite of models for various junction and link types at various levels of detail. These models were designed to relate total accidents, or types of accidents, to an appropriate measure of flow with or without geometric design parameters. The categorisation of accidents differed for junction and link models but examples included shunts, accidents involving a pedestrian, and accidents involving overtaking. These models are widely used in safety appraisal in spite of the age of the modelled data. Thus, for example, in the current version of ARCADY, PAMs for 4-arm roundabouts are based on data for the years 1974-79 (Maycock and Hall 1984). In PICADY the modelled data is for the years 1979-83 for rural 3-arm priority junctions (Pickering et al., 1986) and 1983-89 for 3arm priority junctions in urban areas (Summersgill et al.,1996). Subsequent studies have investigated the relationship between accidents and a wider range of parameters such the time of day (Turner and Thomas, 1986) and congestion (Wang et al., 2009). New models relating accidents to traffic flow and geometric parameters have also been produced (Hashim and Bird, 2005). Nevertheless the TRL models remain the industry standard and updating these models is the focus of the present study. These TRL models pre-date many significant road safety initiatives in the UK including, for example, the announcement of the first National Casualty Reduction target (1987), compulsory use of seatbelts (where fitted) for rear-seat passengers (introduced for children, 1989; adults, 1991), the widespread deployment of speed cameras (first introduced in 1992), the launch of the THINK! road safety campaign (2000) and the widespread introduction of airbags and crumple zones in vehicles. 
Given that the national personal injury accident total fell by $30 \%$ between 1985 and 2009, while over the same period total road traffic increased by 61\% (DfT 2010a; DfT 2010b), PAMs derived using data from some 20 to 30 years ago seem likely to over-estimate expected accidents now. The issue of model ageing is not easily dealt with but equally, without reliable models, errors in scheme appraisal and evaluation seem inevitable. Large, expensive modelling studies of the type carried out by TRL between the 1970s and 1990s are likely to be, at best, only a temporary solution. What is needed is a methodology that will allow existing PAMs to be readily and reliably updated to any point in time.

\subsection{Aims of the study}

The present study, of which this paper is a part, has two principal objectives. Firstly to establish the extent to which the temporal transferability of available PAMs varies with model complexity and, in particular, the extent to which the inclusion of design variables in more complex models increases temporal stability. Secondly to develop an approach that will allow PAMs to be readily and reliably updated to any point in time. To allow these objectives to be achieved a database has been compiled containing accident data, flow data and geometric design parameters for six site categories; modern rural single carriageway A-roads, modern rural dual carriageway Aroads, urban single carriageways, urban 3-arm signalised junctions, urban 4-arm signalised junctions and 4-arm roundabouts. The aim was to include a range of link and junction types and model ages.

The focus of this paper is modern single carriageway A-road links in rural areas which are amongst the most recent of the TRL models. TRL developed models of these roads at four levels of detail (Walmsley et al., 1998): Level 1A models related total accidents to an appropriate measure of flow; level 1B models were similar to level 1A 
but geometric design parameters were included as explanatory variables; level 2 models were similar to level 1A models but accidents were disaggregated by type; level 3 models were also for accidents disaggregated by type but geometric parameters were included. The TRL accident data were collected for the period 1979 to 1990 . The goodness-of-fit of the existing TRL models to recent data was determined. Updating strategies were trialled and new parameter estimates were made using the recent data to assess how these parameters have changed.

\section{Database}

The database complied for this analysis contained 341 minor links distributed amongst 73 schemes. A scheme referred to the largest feature studied. In this paper it was a section of road with similar flow characteristics normally starting and ending at a major junction A major junction was defined as anywhere that traffic on the main road of the scheme had to give way. A minor junction was defined as any other junction which was properly marked with a give way or stop line and a centre line on at least one junction arm. A minor link was the section of road between any two junctions. Typically any given road number (e.g. A14) appeared for only one scheme in the database.

Most of the schemes were analysed across a five year period (2005-2009). However major changes to two schemes meant that shorter study periods of 2005-2007 and 2005-2008 were used. A summary of the schemes contained in the present study (hereafter referred to as the LL or Liverpool-Leeds study) is compared to that used by TRL in Table 1. Although TRL used data collected between 1979 and 1990 individual schemes contained between 1 and 12 years of data with more schemes studied towards the end of the period: $68 \%$ of the accidents in the TRL study occurred between 1986 and 1990. The TRL sample gave an even spread of sites across England with the exception of London as this region is predominantly urban. In the LL study 
approximately $50 \%$ of schemes were within the area covered by Lancashire County Council and the remaining schemes were randomly selected from those used by TRL. An advantage of using a large proportion of schemes from Lancashire was that the accident data were checked rigorously by local authority staff using the text records provided by the police to verify the location of the accident, the severity and the manoeuvres performed by the vehicles. Comparison of the Lancashire County Council data with the national STATS19 database suggested that approximately $4 \%$ of accidents would have been assigned to the wrong link if the national database had been used in Lancashire. The flow data provided by Lancashire County Council often contained several measurements on one major link, allowing the variation due to traffic leaving or joining at minor junctions or accesses to be observed. The schemes selected from the TRL sample used accident data from the STATS19 database with the accidents assigned to the nearest link. Traffic flow data were obtained from the DfT who measure or estimate traffic flow on every major link on A-roads in the UK every year and thus at least one value of traffic flow was available for each link in each year.

[Insert table 1 roughly here, caption follows reference list].

The sample size was smaller in the LL study, containing $60 \%$ of the road length used by TRL. The total number of accidents in the LL study was $71 \%$ of those reported by TRL, although the LL study used 5 years of data (2005-2009) whilst TRL used up to 12 years (1979-1990). The mean annual average daily traffic (AADT) was 35\% larger in the LL study than in the TRL study. This was somewhat less than the increase of $61 \%$ reported nationally across the same period. The accident rates per 100 million vehicle $\mathrm{km}$ were not significantly different in the TRL or LL studies. There was a shift towards less serious accidents in the LL study with an average accident severity of $42 \%$ 
in the TRL study and $26 \%$ in the LL study. (Accident severity is defined here as the ratio of fatal and serious accidents to all accidents expressed in \%). A higher proportion of accidents took place at minor junctions in the TRL study than in the LL study. A possible explanation was the higher minor junction density of $2.7 \pm 0.1$ minor junctions per $\mathrm{km}$ in the TRL study compared to $1.0 \pm 0.1$ minor junctions per $\mathrm{km}$ in the LL study (these errors are the standard error of the sample mean).

TRL measured or derived 35 geometric design parameters for each minor link. In the LL study only those parameters which TRL found to be significant in the models at the $5 \%$ level were recorded in the database. These parameters were:

- Presence or absence of hardstrip on the minor link.

- Width of scheme (classified as "standard" or "wide").

- Hilliness (sum of the height gain \& loss on a link, divided by the link length (m / $\mathrm{km})$ ).

- Bendiness (sum of the angles turned on a link, divided by the link length $\left(^{\circ} /\right.$ $\mathrm{km})$ ).

- Net gradient (height difference between the end points of a link, divided by the link length. (\%)).

These parameters are summarised in Table 2.

[Insert table 2 roughly here, caption follows reference list].

In order to test the full range of TRL models it was necessary to disaggregate accidents by type. The number and proportion of accidents of each type were compared to those reported by TRL in Table 3. A number of accidents in the LL study could be classified as being of more than one type. To prevent double counting of accidents the 
following hierarchy was used for accidents which could be classified into two or more categories:

- Pedestrian accidents.

- Access accidents if any vehicle was turning or waiting to turn.

- Accidents which involved a parked vehicle.

- All other categories.

The $\%$ of total accidents column does not add up to $100 \%$ as accidents at minor junctions were excluded from these categories but not from the total number of accidents. In addition not all link accidents could be assigned to one of the categories. It can be noted in the last column of Table 3, that the change in the accident rate varies with accident type. Whilst the accident rate for most types of accident declined, the rate for shunts and accidents at accesses increased by $49 \%$; even though the number of accidents at accesses remained small ( 84 in 5 years) both of these increases were statistically significant at the $5 \%$ level.

[Insert table 3 roughly here, caption follows reference list].

\section{Goodness of fit of TRL models and updating strategies}

To assess how well, or otherwise, the TRL models fit recent data these models were used to predict the number of accidents on the links in the LL database. The differences between the observed and predicted accident frequencies (accidents per year) were used to calculate the root mean square error (RMSE) and the fit of the model to the data was used to calculate the log likelihood. Lower values of the RMSE indicated closer agreement between the predictions and the observations. If the model with the lowest RMSE contained geometric design features then the log likelihood was used in a likelihood ratio test to establish whether adding extra parameters significantly improved 
the model fit. If the improvement was not significant at the $5 \%$ level then the model with the next lowest RMSE was considered. As these tests were based on values of twice the $\log$ likelihood $2 \log \mathrm{L}$ is given throughout this paper.

The TRL model relating the total accident frequency on a minor link to flow and geometric design parameters is used as an example of how each model was treated. This model was:

$$
A=\exp (-8.037) \cdot Q^{0.827} \cdot L^{0.923} \cdot \exp (-0.423 \cdot H S-0.322 \cdot W S c h e m e)
$$

where A was the annual accident frequency, Q was the annual average daily traffic flow (AADT in vehicles / day) and L was the link length (km). HS was a two-level factor with $\mathrm{HS}=1$ where hardstrips were present and $\mathrm{HS}=0$ where hardstrips were absent. WScheme was a two-level factor with WScheme $=0$ for standard width roads and WScheme $=1$ for wider roads. Accident frequencies were predicted in each of the five years and the total predictions are compared to the observed values. The results are shown in Figure 1. The top left panel shows the observed average annual accident frequency (y-axis) as a function of predicted annual accident frequency ( $\mathrm{x}$-axis) and the solid line indicates the line where observed and predicted accident frequencies are equal. This panel show a relationship between the predicted and observed values, albeit with a substantial amount of scatter. The RMSE was 0.558 and $2 \log L$ was -1339 . The residual difference between the observed and predicted accident frequencies is shown in the top right panel of Figure 1. The solid line indicates a residual of zero and the dashed lines indicate residuals of 1 and -1 . The striped appearance of this plot arose as the observed number of accidents can only take integer values. The model tended to underestimate the accident frequencies: it under estimated the number of accidents on 238 minor links and over estimated on 103 minor links. However the magnitude of the 
over predictions tended to be larger than the under predictions. In total 996 accidents were observed and 1252 accidents were predicted.

[Insert figure 1 roughly here, caption follows reference list].

The large number of minor links with small numbers of accidents meant that numerous links were plotted in a small area of the lowest left panel of Figure 1. To enhance the clarity of this plot and to establish whether there was an underlying trend in the mean value these data were combined; the predicted values were ranked in ascending order and grouped into bins of ten sites by taking the lowest ten ranked sites, the next ten and so on. The mean predicted, mean observed and residual values for each bin were then plotted in the lower panels of Figure 1. These lower panels suggest that there is an underlying trend in the mean value.

There were several possible updating strategies for this model. A year-on-year change in accident frequency could be incorporated into the PAM as a multiplicative trend term of the form $\exp (\theta \cdot t)$ where $\theta$ is the annual trend and $t$ is the number of years since 1990, the year for which the model was developed. This was considered but, since there was no significant trend (at the $5 \%$ level) in the TRL study, this was not a suitable updating strategy. It is also possible to apply a trend based on national data (DfT, 2010b) for rural A-class roads although available national data includes both single and dual carriageways and traditional and modern standard roads whereas in the present study the data includes only modern single carriageway roads. A national trend of $-0.92 \%$ p.a. was established but when this trend was applied, the model fit was worse than the fit without correction. A third updating strategy was to use the recent data to calibrate the original TRL models. The observed and predicted accident frequencies were used to establish a scaling factor, $s f$. 


$$
s f=\frac{\sum_{i=1}^{n} \text { observed }_{i}}{\sum_{i=1}^{n} \text { predicted }_{1}}
$$

where observed is the number of accidents observed on each minor link in the study period, predicted is the number of accidents predicted on each minor link in the study period and $n$ is the number of minor links. The use of this scaling factor minimised the absolute mean error (AME). Predictions for individual minor links were then simply obtained by multiplying the TRL model estimates by the scaling factor. In the case of the model for total accidents on minor links (equation 1), the mean number of accidents observed per year was $0.58( \pm-0.04)$ and the mean number predicted using equation 1 was $0.73( \pm-0.03)$ where the uncertainties are the standard error of the parameter

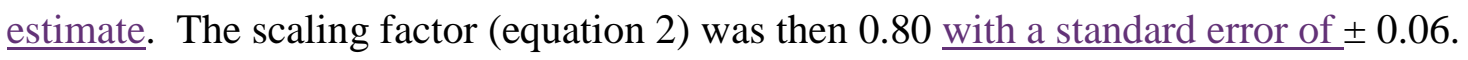
When this scaling factor was applied the RMSE was reduced from 0.558 to 0.526 and $2 \log \mathrm{L}$ increased from -1339 to -1318 . Also the model did not show the same tendency to over predict: over predictions occurred on 140 minor links and under prediction occurred on 201 minor links.

There were 13 minor links where the residual was greater than one accident per year. These minor links had a significantly longer length with a mean value of 1.7 $( \pm 0.3) \mathrm{km}$ compared to the mean value for the entire database of $0.89( \pm 0.04) \mathrm{km}$ where the uncertainties are the standard error of the sample mean. However, although these links were longer on average, not all longer links had larger residuals. There was no other significant relationship between the residuals and any of the geometric design parameters or flow.

This process was repeated for each TRL model in turn and the results are presented in Table 4. The 'TRL model' columns show the RMSE and $2 \log L$ for the 
models with and without geometric design parameters included as factors. The 'calibration' columns show the scaling factor (SF), the standard error (SE) in this value, the RMSE and $2 \log L$ for the model fit. The calibrated model of total accidents with geometric design parameters had a slightly lower RMSE than the model of total accidents without geometric design parameters, although this difference was small. The log likelihood showed very little change and likelihood ratio tests showed that the addition of the geometric parameters did not significantly improve the model fit at the 5 $\%$ level. For almost all models of accident types TRL presented the best fitting model without geometric factors and, if additional geometric factors improved the model fit, the best fitting model with factors. Therefore the models with geometric factors gave a better fit than those without these terms in the TRL study. In the present study models of accidents disaggregated by type tended to give a slightly better fit when geometric design parameters were excluded. The goodness-of-fit for models of different types of accident cannot be directly compared in Table 4 as this does not account for the different number of accidents of each type. To compare whether the total accident or the accident type models better represented the data, the predictions from the accident type models were summed and compared to the observed total accident frequency. Not all of the link accidents were assigned to one of the accident types, therefore the summed predictions had to be rescaled by multiplication by a factor of 1.14 . The results are shown in Table 5. These show that the model of total accidents gave a better fit to the data than those disaggregated by accident type.

[Insert tables 4 and 5 roughly here, captions follows reference list].

Calibration was attempted for each TRL model. The scaling factors and the goodness-of-fit of the models with and without calibration are shown in Table 4. The 
scaling factors were directly applied to the TRL models for example the unscaled model for shunts was:

$$
A=\exp (-17.19) \cdot Q^{1.51} \cdot L^{0.612}
$$

where A was the annual accident frequency, Q was the annual average daily traffic flow (AADT in vehicles / day) and L was the link length $(\mathrm{km})$. The calibrated model was:

$$
A=1.58 \cdot \exp (-17.19) \cdot Q^{1.51} \cdot L^{0.612}
$$

The small number of accidents in some of the accident type categories meant that care had to be taken when calibrating these models. The approach chosen was to use one scaling factor for all the accident types in which the accident rate had fallen and to calculate separate scaling factors for 'access' and 'shunt' accidents as these rates had increased. Different scaling factors were calculated for models with and without geometric parameters to establish whether the inclusion of these parameters affected the temporal transferability of the models. The scaling factors for the accident type models were: 1.58 for 'shunts, without geometric factors'; 3.18 for 'access, without geometric factors'; 3.33 for 'access, with geometric factors'; 0.71 for all other accident types without geometric factors and 0.73 for all other accident types with geometric factors. There was no model for shunts with geometric factors. The use of a scaling factor improved the model fit (given by $2 \log L$ ) in all cases with the exception of 'overtake, same direction', although in some cases this change was small. The addition of geometric parameters to the models did not significantly improve the fit of the model to the data. The predictions for the calibrated accident type models were summed and compared to the observed total accident frequencies. The RMSE (Table 5) show that 
calibration improved the model fits to the data although in most cases this improvement was small.

\section{New parameter estimates}

The next step was to investigate whether the relationship between accidents and the various explanatory variables had changed over time. New parameter estimates were found by re-fitting the models using our database. These models had the same form and included the same variables and factors as those given by TRL. To distinguish these models from the original TRL models, these are referred to as the LL model fits. As the purpose of fitting these models was to establish how the parameter estimates in the TRL models have changed over time, only models of the same form as those given by TRL were tried. The number of accidents in a given period at a given site followed a Poisson distribution about the site mean. However, as there was considerable variation in these site means, these data were overdispersed. Conventionally it is assumed that the overdispersion is gamma distributed about the site mean (see, for example, Maher and Summersgill, 1996), so that the combined distribution is negative binomial (NB) and this was used in the present study.

The results of the re-fitting are shown in Table $6 \mathrm{a}$ and the overdispersion parameters are shown in Table 6b. The models of total accidents are shown together with the best fitting model for each type of accident. These model fits showed that it was rare for a new parameter estimate to be significantly different from the value given by TRL. Notable exceptions were the traffic flow (AADT) in the 'single vehicle, vehicle did not leave carriageway' and 'shunts' models and the link length in the 'overtake, same direction' and 'head on' models. In many of the accident type models either traffic flow 
or link length were not significant parameters and geometric factors were only significant in one of the accident type models (head on). This was in part a consequence of the large standard errors arising from the small number of accidents of each type. The lack of significant geometric factors for many of the accident type models was expected as the original TRL models without geometric factors gave a better fit to the LL database. This may be a consequence of over fitting in the original TRL models. The usefulness of models which do not contain both flow and link length is somewhat questionable: these model fits do not suggest that the accident frequency is independent of these parameters, they merely show that there is no relationship that is statistically significant at the 5\% level. The model of 'Single vehicle, did not leave carriageway' had a negative power of flow suggesting that, as traffic flow increased, accidents of this type decreased. This is not unreasonable as, on higher flow roads, these may have become multi-vehicle accidents. However, since there were only 23 accidents of this type in the LL database, additional data would be needed to confirm this relationship. It was expected that the LL models would give a better fit to the LL database than the TRL models as the LL models were developed from these data. In general the LL models where the accidents were disaggregated by accident type gave a slightly better fit than the original TRL models. In the case of the models of total accidents, the new parameter estimates did present a more substantial improvement. The overdispersion parameters were somewhat larger for the LL models than the TRL models although, in general, this increase was not statistically significant at the $5 \%$ level (a larger value of the overdispersion parameter means less dispersion). The overdispersion parameters for the calibrated models are also shown; in general these values were larger than those for the TRL models but smaller than those for the LL models. 
[Insert tables $6 \underline{a \text { and } b}$ roughly here, caption follows reference list].

Predictions from the disaggregated models were combined and compared to the total accident frequency to determine whether the accident type or the total accidents models better represented the data. The method used was the same as that described in Section 3 and the results are shown in Table 7. As geometric design parameters were only significant in one of the accident type models the disaggregated models with geometric design parameters were not included. The best fitting model was the model of total accidents with geometric design parameters but this model was not significantly better than the model without these parameters. The models of total accidents gave a better fit to the data than models disaggregated by accident type.

[Insert table 7 roughly here, caption follows reference list].

Since approximately half of the schemes used in this analysis came from the small geographic region covered by Lancashire County Council it was important to establish whether there was any evidence that this area differed from the rest of the UK. New parameter estimates were made for the models of total accidents, firstly using only data from within the Lancashire County Council area and secondly using only data from outside this area. These estimates were not significantly different (at the $5 \%$ level) from those produced from the study as a whole.

\section{Discussion}

The national personal injury accident total fell by $30 \%$ between 1985 and 2009, while over the same period total road traffic increased by 61\% (DfT 2010a; DfT 2010b). These figures imply that the accident rate fell by $\sim 50 \%$ and so it would appear reasonable to expect the PAMs developed by TRL based on data for the period 19861990 to over-estimate expected accidents. The PAMs of total accidents required calibration with a scaling factor of 0.91 for the model without geometric factors and 
0.80 for the model with geometric factors. These factors were rather larger than might be expected on the basis of the national data. One possible explanation was that the national statistics include all types of road and the proportion of modern standard roads in this sample increased over time, whereas the data used to develop the PAMs were for modern standard roads only. It was likely that part of the reduction in national accident rate is because new roads are constructed to a higher design standard. Another possible explanation was that the changes in accident rates were different for different types of accidents. Whilst the models of most types of accident (without geometric design features) required a scaling factor of 0.71 , the values for accidents at accesses and shunts were 3.18 and 1.58 respectively. It could be that the downward trend for most types of accident is in line with national statistics but that these reductions have been offset by the increasing number of accidents at accesses and shunts.

The mean traffic flow was 35\% greater in the LL study than in the TRL study although the accident rates were not significantly different. The use of accident rates, of course, had limitations in that it assumed that the accident frequency increased linearly with traffic flow whereas in the TRL models the accident frequency was a sub-linear function of traffic flow. This meant that, even in the absence of a trend in accidents, the increase in traffic flow would decrease the accident rate. In the present study this reduction would be about $5 \%$ but the uncertainty in the estimate of the accident rate meant that this would not be significant at the 5\% level. The use of a national trend in accidents did not improve the model fit although other methods to determine a trend for these types of roads may be more appropriate and will be discussed in detail in a future publication.

The accident severity was somewhat lower in the LL study with a mean value of $25 \%$, compared to the value of $42 \%$ stated by TRL. Several major road safety initiatives 
have been undertaken in the UK in the intervening period which may account for this. These include the announcement of the first National Casualty Reduction target (1987), compulsory use of seatbelts (where fitted) for rear-seat passengers (introduced for children, 1989; adults, 1991), the launch of the Think! road safety campaign (2000) and the widespread introduction of airbags and crumple zones in vehicles.

The PAMs for total accidents provided a remarkably good fit to the recent data. This fit was improved by calibration. The more complex model, which included geometric design parameters, did not give a significantly better fit to the data than the model without these features. The models of total accidents gave a better fit to the data than combining the predictions from the accident type models. New parameter estimates improved the predictions for the models of total accidents. However this improvement needs to be balanced against the cost of the detailed database which is required to achieve it. Calibration of existing models thus seems likely to be a more a cost effective option.

It should be noted that these results are for modern rural single carriageway Aroads links only. Other site types may give different results. Indeed preliminary results for modern rural dual carriageway A-roads show that the accident rate has fallen significantly and indicate that a trend should be allowed for within the models. Studies are also in progress to test models which include links and minor junctions together for both single and dual carriageway modern rural roads.

\section{Conclusion}

Reliable predictive accident models (PAMs) are essential to design and maintain safe road networks and yet the models in current use were derived using data collected 20 to 30 years ago. Despite the age of these models the PAMs for total accidents on rural single carriageway A-roads provided a remarkably good fit to the recent data and 
calibration was found to improve the model fit. The model which gave the best fit to the data excluded geometric design parameters and required a scaling factor of 0.91 . This model provided a better fit to the current data that the models which predicted accidents by type. When accidents were disaggregated by accident type, the addition of geometric design parameters in these models did not improve the model fit. The proportions of the various types of accident have changed significantly over time and a calibration of these models against recent data improved the fit with scaling factors of 1.58 for shunts, 3.18 for access accidents and 0.71 for all other accident types.

These models for modern, rural, A-class roads do not over predict accidents to the extent that might be expected given the national changes in the national personal injury accident total and the traffic flow. However, the national statistics include all types of road whereas the PAMs were developed by TRL for modern standard roads only. It is likely that the proportion of roads designed to modern standards has increased over time and so, at least in part, the national reduction in accident rate is as a result of a higher proportion of modern standard roads.

Acknowledgements.

Financial support for this paper was provided by the UK Engineering and Physical Sciences Research Council under grant EP/H046739/1 and the project partners were Lancashire County Council (LCC). The authors would like to thank Peter Andrews, David Cronshaw, Nigel Cleave, Richard Donkin, Ashley Newnham and their colleagues in the Environment Directorate, LCC for their extensive assistance in compiling the data for this paper. Also gratefully acknowledged are the contributions of Blackburn with Darwen Borough Council, Blackpool Council, Cheshire West and Cheshire Council, Derbyshire County Council, Hampshire County Council, Leeds City Council, Leicestershire County Council, Lincolnshire County Council, Kirklees Council, North Yorkshire County Council, Northamptonshire County Council, Nottinghamshire County Council, Staffordshire County Council, Transport for Greater Manchester (formerly GMTU) and Warwickshire County Council. The figures for this paper were produced using tools developed by Karl Ropkins from the University of Leeds. 
References:

DfT (2009) Transport Analysis Guidance. http://www.dft.gov.uk/webtag/

DfT (2010a) Road Casualties Great Britain: 2009. Department for Transport, London.

DfT (2010b) Transport Trends 2009. Department for Transport, London.

Elvik, R. (2008) The predictive validity of empirical Bayes estimates of road safety. Accident Analysis \& Prevention, 40, 1964-1969.

Hashim I. H. and Bird R. N. (2005), Analysis of Accident Rates and Geometric Consistency Measures on Sections of Rural Single Carriageway, Road Safety on Four Continents (VTI/TRB Conference), Warsaw, Poland.

Hauer, E. (2004) Statistical road safety modelling. Transportation Research Record 1897, 81-87.

Maher, M. J. and Mountain, L. J. (2009) The sensitivity of estimates of regression to the mean. Accident Analysis and Prevention 41, 861-868.

Maher M. J. and I. Summersgill A comprehensive methodology for the fitting of predictive accident models. Accident Analysis and Prevention 28(3), 1996, 281296. Maycock G. , R. D. Hall (1984) Accidents at four-arm roundabouts. LR1120, TRL, Crowthorne, UK.

Mountain, L. J., Hirst, W. M. and Maher, M. J. (2005) Are speed enforcement cameras more effective than other speed management measures? The impact of speed management schemes on 30mph roads. Accident Analysis and Prevention 37, $742-752$.

Persaud, B, C. Lyon (2007) Empirical Bayes before-after safety studies: Lessons learned from two decades of experience and future directions. Accident Analysis and Prevention 39, 546-555.

Pickering, D., Hall, R., and Grimmer, M. (1986) Accidents at rural T-junctions. TRL Research Report 65, TRL, Crowthorne, UK.

Turner, D. J. And Thomas, R. (1996), Motorway accidents: an examination of accident totals, rates and severity and their relationship with traffic flow. Traffic Engineering and Control 27 (7/8).

Summersgill, I., Kennedy, J., and Barnes, D. (1996) Accidents at 3-arm priority junctions on urban single-carriageway roads. TRL Report 184, TRL, Crowthorne, UK.

Walmsley, D.A., Summersgill, I. and Binch, C. (1998) Accidents on modern rural single-carriageway trunk roads, Transport Research Laboratory Report 336. 
Wang, C., Quddus, M. A. and Ison, S. G., 2009. Impact of traffic congestion on road accidents: a spatial analysis of the M25 motorway in England. Accident Analysis \& Prevention, 41(4), pp. 798-808.

Page 22 


\begin{tabular}{|c|c|c|c|}
\hline \multicolumn{2}{|c|}{ Summary variables } & TRL & \multirow[t]{2}{*}{$\mathbf{L L}$} \\
\hline \multicolumn{2}{|c|}{ Scheme length $(\mathrm{km})$} & & \\
\hline \multicolumn{2}{|c|}{ Total length of schemes } & 540.5 & 323.5 \\
\hline \multicolumn{4}{|c|}{ Link length (km) } \\
\hline & Mean & & 0.9 \\
\hline & Maximum & & 3.9 \\
\hline & Minimum & & 0.01 \\
\hline & Standard Deviation & & 0.7 \\
\hline \multicolumn{4}{|c|}{ Accidents } \\
\hline \multicolumn{2}{|c|}{ Total number of accidents } & 2111 & 1494 \\
\hline \multirow[t]{6}{*}{ of which: } & Fatal & 180 & 77 \\
\hline & Serious & 700 & 307 \\
\hline & Slight & 1231 & 1110 \\
\hline & Minor Junction & 816 & 498 \\
\hline & Minor Link & 1295 & 996 \\
\hline & Av. Acc. Sev. & $42 \%$ & $26 \%$ \\
\hline \multicolumn{4}{|c|}{ Flow (AADT) } \\
\hline & Mean & 10309 & 13878 \\
\hline & Minimum & 2883 & 2887 \\
\hline & Maximum & 35904 & 31812 \\
\hline & Standard Deviation & & 5784 \\
\hline \multicolumn{4}{|c|}{ Accident rate (accidents / 100MVehkm) } \\
\hline & Mean & 19.7 & 19 \\
\hline & Maximum & 68.7 & 56.6 \\
\hline & Minimum & 2.0 & 0.0 \\
\hline & Standard Deviation & & 24 \\
\hline
\end{tabular}

Table 1. Comparison of sample selected in the TRL study and the LL study. Empty cells in the TRL column indicate that data were not available. 


\begin{tabular}{lccc} 
Summary variables & $\begin{array}{c}\text { Bendiness }(\mathbf{d e g} / \\
\mathbf{k m})\end{array}$ & Hilliness $(\mathbf{m} / \mathbf{~ k m})$ & Net Gradient \\
\hline Mean & 45.3 & 20.6 & $1.6 \%$ \\
Maximum & 645.2 & 81.2 & $8.1 \%$ \\
Minimum & 0.0 & 0.0 & $0.0 \%$ \\
Standard Deviation & 58.0 & 13.7 & $1.5 \%$
\end{tabular}

Table 2a. Comparison of geometric variables in the TRL study and the LL study.

\begin{tabular}{|lcc|}
\hline Summary factor & Hardstrip & Width of scheme \\
\hline Level 0 & 200 & 69 \\
Level 1 & 141 & 4
\end{tabular}

Table 2b. Comparison of geometric factors in the TRL study and the LL study. 


\begin{tabular}{|c|c|c|c|c|c|c|c|}
\hline \multirow[b]{2}{*}{ Accident Type } & \multicolumn{3}{|c|}{ TRL study } & \multicolumn{3}{|c|}{ LL study } & \multirow{2}{*}{$\begin{array}{c}\text { Change } \\
\text { Acc. } \\
\text { rate }\end{array}$} \\
\hline & Acc. & $\begin{array}{l}\% \text { of } \\
\text { acc. }\end{array}$ & $\begin{array}{l}\text { Acc. } \\
\text { rate }\end{array}$ & Acc. & $\begin{array}{l}\% \text { of } \\
\text { acc. }\end{array}$ & $\begin{array}{l}\text { Acc. } \\
\text { rate }\end{array}$ & \\
\hline 1. Pedestrian & 60 & $2.8 \%$ & 0.85 & 17 & $1.1 \%$ & 0.22 & $-75 \%$ \\
\hline 2. Access & 51 & $2.4 \%$ & 0.72 & 84 & $5.6 \%$ & 1.07 & $49 \%$ \\
\hline 3. 1 veh, nearside & 134 & $6.3 \%$ & 1.89 & 65 & $4.4 \%$ & 0.83 & $-56 \%$ \\
\hline 4. 1 veh, offside & 80 & $3.8 \%$ & 1.13 & 43 & $2.9 \%$ & 0.55 & $-52 \%$ \\
\hline 5. 1 veh, on carriageway & 59 & $2.8 \%$ & 0.83 & 23 & $1.5 \%$ & 0.3 & $-65 \%$ \\
\hline 6. Parked & 37 & $1.8 \%$ & 0.52 & 32 & $2.1 \%$ & 0.41 & $-22 \%$ \\
\hline 7. Overtake, same direction & 83 & $3.9 \%$ & 1.17 & 57 & $3.8 \%$ & 0.73 & $-38 \%$ \\
\hline 8. Overtake, head on & 136 & $6.4 \%$ & 1.92 & 67 & $4.5 \%$ & 0.85 & $-77 \%$ \\
\hline 9. Overtake, other collision & 128 & $6.1 \%$ & 1.81 & * & * & * & $*$ \\
\hline 10. Shunt & 182 & $8.6 \%$ & 2.57 & 301 & $20.2 \%$ & 3.83 & $49 \%$ \\
\hline 11. Head on & 279 & $13.2 \%$ & 3.94 & 183 & $12.2 \%$ & 2.33 & $-41 \%$ \\
\hline
\end{tabular}

The accident rate is the number of accidents per 100 Mvehkm.

The full description of each category of accident is: 1. Pedestrian accidents. 2. Accidents at accesses. 3. Single vehicle, left carriageway on nearside on a straight road. 4. Single vehicle, left carriageway on offside on a straight road. 5. Single vehicle, did not leave carriageway. 6 . Accident involving a parked vehicle. 7. 2+ vehicles, overtaking, same direction. 8. 2+ vehicles, overtaking, opposite direction, headon. 9. 2+ vehicles, overtaking, opposite direction, other collision. 10. 2+ vehicles, no overtaking, same direction. 11. 2+ vehicles, no overtaking, opposite direction.

* As the current STATS19 fields meant that the 'overtake, head on' and 'overtake, other collision' accident types could not be reliably separated these were combined into one group called 'overtake, opposite direction' in the LL study.

Table 3. Breakdown of accidents by type in the TRL and LL studies. 


\begin{tabular}{|c|c|c|c|c|c|c|c|}
\hline \multirow[t]{2}{*}{ Model } & \multirow{2}{*}{$\begin{array}{l}\text { With } \\
\text { geom. }\end{array}$} & \multicolumn{2}{|c|}{ TRL model } & \multicolumn{4}{|c|}{ Calibration } \\
\hline & & RMSE & $2 \log L$ & SF & SE & RMSE & $2 \log L$ \\
\hline All accidents & No & 0.531 & -1323 & 0.91 & 0.07 & 0.529 & -1318 \\
\hline All accidents & Yes & 0.558 & -1339 & 0.8 & 0.06 & 0.526 & -1318 \\
\hline Pedestrian & No & 0.032 & -173 & 0.73 & 0.05 & 0.031 & -166 \\
\hline Pedestrian & Yes & 0.036 & -173 & 0.71 & 0.05 & 0.034 & -166 \\
\hline Access & No & 0.090 & -569 & 3.18 & 0.43 & 0.088 & -511 \\
\hline Access & Yes & 0.090 & -572 & 3.33 & 0.45 & 0.088 & -510 \\
\hline 1 veh, nearside & No & 0.069 & -432 & 0.73 & 0.05 & 0.067 & -428 \\
\hline 1 veh, nearside & Yes & 0.073 & -448 & 0.71 & 0.05 & 0.070 & -447 \\
\hline 1 veh, offside & No & 0.041 & -315 & 0.73 & 0.05 & 0.040 & -313 \\
\hline 1 veh, offside & Yes & 0.051 & -324 & 0.71 & 0.05 & 0.051 & -321 \\
\hline 1 veh, on carriageway & No & 0.047 & -215 & 0.73 & 0.05 & 0.046 & -213 \\
\hline Parked & No & 0.054 & -276 & 0.73 & 0.05 & 0.050 & -280 \\
\hline Overtake, same direction & Yes & 0.064 & -394 & 0.71 & 0.05 & 0.064 & -398 \\
\hline Overtake, opposite direction & No & 0.048 & -479 & 0.73 & 0.05 & 0.046 & -461 \\
\hline Shunt & No & 0.197 & -1191 & 1.58 & 0.15 & 0.195 & -1152 \\
\hline Head on & No & 0.108 & -869 & 0.73 & 0.05 & 0.108 & -884 \\
\hline
\end{tabular}

Table 4. Goodness of fit of the TRL PAMs to the LL database with and without geometric factors. 


\begin{tabular}{|lcccc|}
\hline \multirow{2}{*}{ TRL model } & \multicolumn{2}{c}{ RMSE } & \multicolumn{2}{c|}{ 2logL } \\
& $\begin{array}{c}\text { No } \\
\text { geom. }\end{array}$ & $\begin{array}{c}\text { With } \\
\text { geom. }\end{array}$ & $\begin{array}{c}\text { No } \\
\text { geom. }\end{array}$ & $\begin{array}{c}\text { With } \\
\text { geom. }\end{array}$ \\
\hline All accidents, unscaled & 0.531 & 0.558 & -1323 & -1339 \\
\hline All accidents, scaled & 0.529 & 0.526 & -1318 & -1318 \\
\hline By accident type, unscaled & 0.755 & 0.755 & -2081 & -2080 \\
\hline By accident type, scaled & 0.686 & 0.746 & -2060 & -2059 \\
\hline
\end{tabular}

Table 5. RMSE and likelihood indicating the goodness of fit of different types of TRL model when predictions from the models disaggregated by accident type were combined. 


\begin{tabular}{|c|c|c|c|c|c|c|c|c|c|c|c|c|c|c|c|c|}
\hline \multirow{2}{*}{ Model } & \multicolumn{2}{|c|}{ RMSE } & \multicolumn{2}{|c|}{$2 \log L$} & \multicolumn{3}{|c|}{ Intercept } & \multicolumn{3}{|c|}{ Flow } & \multicolumn{3}{|c|}{ Length } & \multicolumn{3}{|c|}{ HS or Up-Hilliness } \\
\hline & TRL & LL & TRL & LL & TRL & LL & SE & TRL & LL & SE & TRL & LL & SE & TRL & LL & SE \\
\hline All accidents & 0.531 & 0.379 & -1322 & -1291 & -8.407 & -7.305 & 1.436 & 0.833 & 0.724 & 0.118 & 0.865 & 0.925 & 0.067 & & & \\
\hline $\begin{array}{l}\text { All accidents, with } \\
\text { geom. }\end{array}$ & 0.558 & 0.365 & -1338 & -1286 & -8.037 & -7.556 & 1.425 & 0.827 & 0.760 & 0.119 & 0.923 & 0.941 & 0.067 & -0.438 & -0.22 & 0.1 \\
\hline Pedestrian & 0.032 & & -173 & & -11.73 & & & 0.777 & & & 0.687 & & & & & \\
\hline Access & 0.090 & 0.088 & -569 & -507 & -2.73 & -3.58 & 0.251 & -0.217 & & & 0.25 & 0.452 & 0.16 & & & \\
\hline 1 veh, nearside & 0.069 & 0.068 & -432 & -424 & -10.71 & -3.83 & 0.238 & 0.756 & & & 0.944 & 0.627 & 0.179 & & & \\
\hline 1 veh, offside & 0.041 & 0.047 & -315 & -314 & -9.62 & -4.241 & 0.247 & 0.592 & & & 0.655 & 0.638 & 0.204 & & & \\
\hline $\begin{array}{l}1 \text { veh, on } \\
\text { carriageway }\end{array}$ & 0.047 & 0.039 & -215 & -195 & -13.45 & 6.911 & 3.697 & 0.972 & -1.28 & 0.497 & 0.836 & & & & & \\
\hline Parked & 0.054 & & -276 & & -18.67 & & & 1.47 & & & 1.124 & & & & & \\
\hline $\begin{array}{l}\text { Overtake, same } \\
\text { direction }\end{array}$ & 0.064 & 0.064 & -394 & -385 & -9.71 & -12.347 & 4.062 & 0.595 & 0.863 & 0.369 & 0.908 & 0.346 & 0.176 & 0.0225 & 0.0212 & 0.0115 \\
\hline Overtake, head on & & & & & -11.39 & & & 0.834 & & & 0.911 & & & & & \\
\hline $\begin{array}{l}\text { Overtake, other } \\
\text { collision }\end{array}$ & & & & & -9.35 & & & 0.582 & & & 1.254 & & & & & \\
\hline $\begin{array}{l}\text { Overtake, opposite } \\
\text { direction }\end{array}$ & 0.048 & 0.065 & -479 & -436 & & -10.21 & 3.585 & & 0.675 & 0.317 & & 0.493 & 0.165 & & & \\
\hline Shunt & 0.197 & 0.02 & -1191 & -1139 & -17.19 & -11.462 & 2.15 & 1.51 & 0.961 & 0.194 & 0.612 & 0.432 & 0.093 & & & \\
\hline Head on & 0.108 & 0.011 & -869 & -845 & -10.55 & -10.101 & 2.133 & 0.814 & 0.769 & 0.188 & 1.023 & 0.516 & 0.099 & & & \\
\hline
\end{tabular}

The final column is presence or absence of hardstrip for the model of 'all accidents' and up-hilliness for the model of 'overtake, same direction'.

Table 6a. Parameter estimates for TRL PAMs and new LL model fits. The standard errors (SE) of the parameters in the LL models are stated and, if the parameters are significantly different from the TRL estimates at the $5 \%$ level, these are highlighted. 


\begin{tabular}{|c|c|c|c|c|c|c|}
\hline \multirow[t]{2}{*}{ Model } & \multicolumn{2}{|c|}{$\underline{T R L}$} & \multicolumn{2}{|c|}{ Calibration } & \multicolumn{2}{|c|}{ LL model fits } \\
\hline & ODP & SE & ODP & $\underline{\text { SE }}$ & ODP & $\underline{\mathrm{SE}}$ \\
\hline All accidents & 2.68 & 0.47 & 2.78 & $\underline{0.49}$ & 2.85 & $\underline{0.52}$ \\
\hline$\frac{\text { All accidents, with }}{\text { geom. }}$ & $\underline{2.32}$ & $\underline{0.37}$ & $\underline{2.74}$ & $\underline{0.49}$ & $\underline{2.95}$ & $\underline{0.54}$ \\
\hline Pedestrian & $\underline{0.097}$ & $\underline{0.085}$ & $\underline{120}$ & $\underline{2854}$ & $\underline{482}$ & $\underline{8457}$ \\
\hline$\underline{\text { Access }}$ & $\underline{0.12}$ & $\underline{0.03}$ & $\underline{0.24}$ & $\underline{0.08}$ & $\underline{0.24}$ & $\underline{0.08}$ \\
\hline 1 veh, nearside & $\underline{0.36}$ & $\underline{0.16}$ & $\underline{0.46}$ & $\underline{0.24}$ & $\underline{0.51}$ & $\underline{0.28}$ \\
\hline 1 veh, offside & $\underline{5.1}$ & $\underline{22}$ & 8.4 & $\underline{59}$ & $\underline{9.7}$ & $\underline{77.0}$ \\
\hline $\begin{array}{l}\frac{1 \text { veh, on }}{\text { carriageway }} \\
\underline{4}\end{array}$ & $\underline{0.058}$ & $\underline{0.029}$ & $\underline{0.08}$ & $\underline{0.05}$ & $\underline{0.50}$ & $\underline{0.59}$ \\
\hline Parked & $\underline{0.18}$ & $\underline{0.12}$ & $\underline{0.20}$ & $\underline{0.15}$ & $\underline{0.34}$ & $\underline{0.28}$ \\
\hline $\begin{array}{l}\text { Overtake, same } \\
\text { direction }\end{array}$ & $\underline{0.30}$ & $\underline{0.14}$ & $\underline{0.31}$ & $\underline{0.15}$ & $\underline{0.40}$ & $\underline{0.22}$ \\
\hline $\begin{array}{l}\text { Overtake, } \\
\text { opposite direction }\end{array}$ & $\underline{0.17}$ & $\underline{0.05}$ & $\underline{0.30}$ & $\underline{0.11}$ & $\underline{0.88}$ & $\underline{0.64}$ \\
\hline Shunt & $\underline{0.51}$ & $\underline{0.09}$ & $\underline{0.60}$ & $\underline{0.11}$ & $\underline{0.62}$ & $\underline{0.11}$ \\
\hline Head on & $\underline{4.4}$ & $\underline{4.2}$ & $\underline{3.8}$ & $\underline{3.3}$ & $\underline{4.2}$ & $\underline{3.7}$ \\
\hline Accident type & $\underline{1.1}$ & $\underline{0.1}$ & 1.2 & $\underline{0.2}$ & $\underline{1.2}$ & $\underline{0.2}$ \\
\hline
\end{tabular}

$\underline{\mathrm{ODP}}$ is the overdispersion parameter and $\mathrm{SE}$ is the standard error in this value.

$\underline{\text { Table 6b. Overdispersion parameter estimates for TRL PAMs and new LL model fits. }}$ 


\begin{tabular}{|lcccc|}
\hline \multirow{2}{*}{ TRL model } & \multicolumn{2}{c}{ RMSE } & \multicolumn{2}{c|}{ 2logL } \\
& $\begin{array}{c}\text { No } \\
\text { geom. }\end{array}$ & $\begin{array}{c}\text { With } \\
\text { geom. }\end{array}$ & $\begin{array}{c}\text { No } \\
\text { geom. }\end{array}$ & $\begin{array}{c}\text { With } \\
\text { geom. }\end{array}$ \\
\hline All accidents & 0.379 & 0.365 & -1291 & -1286 \\
By accident type & 0.753 & N/A & -2041 & N/A \\
\hline
\end{tabular}

Table 7. RMSE and likelihood indicating the goodness of fit of different types of model using new parameter estimates for the TRL models. 
Rural Single Carriageways: All Data

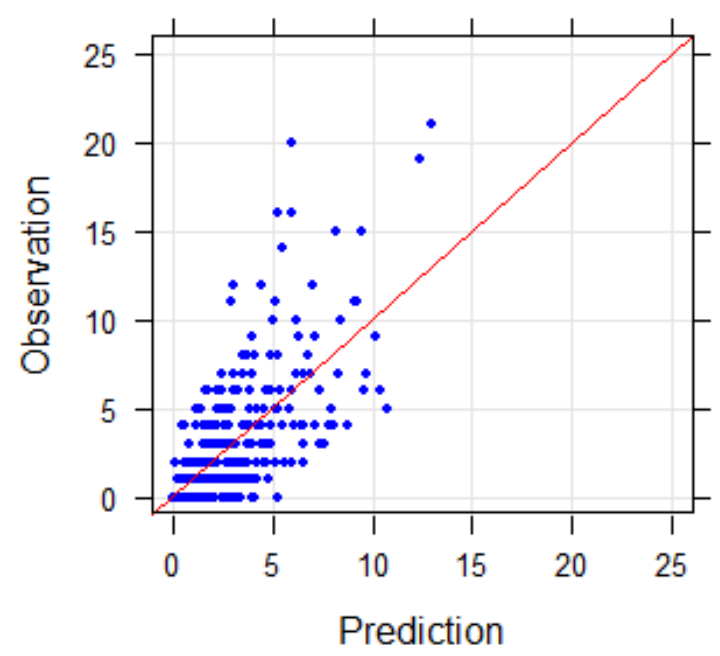

Rural Single Carriageways: Combined Data

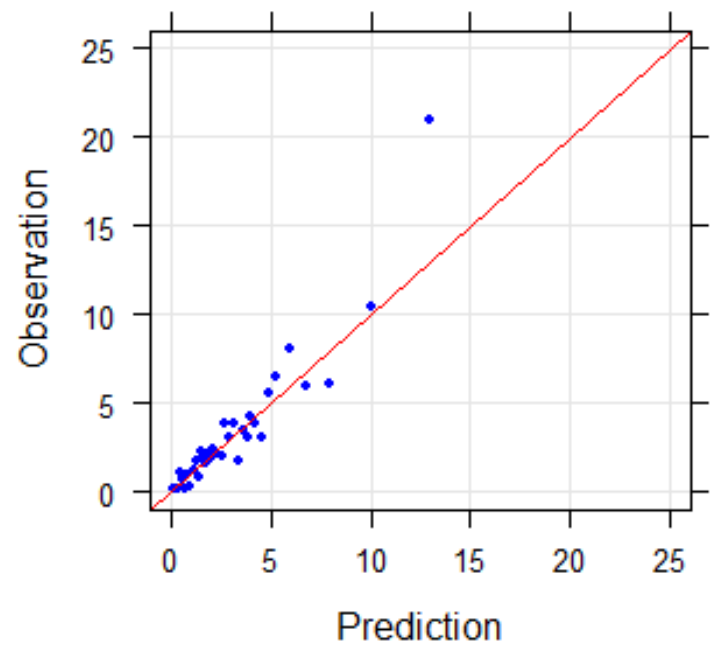

Rural Single Carriageways: Residuals

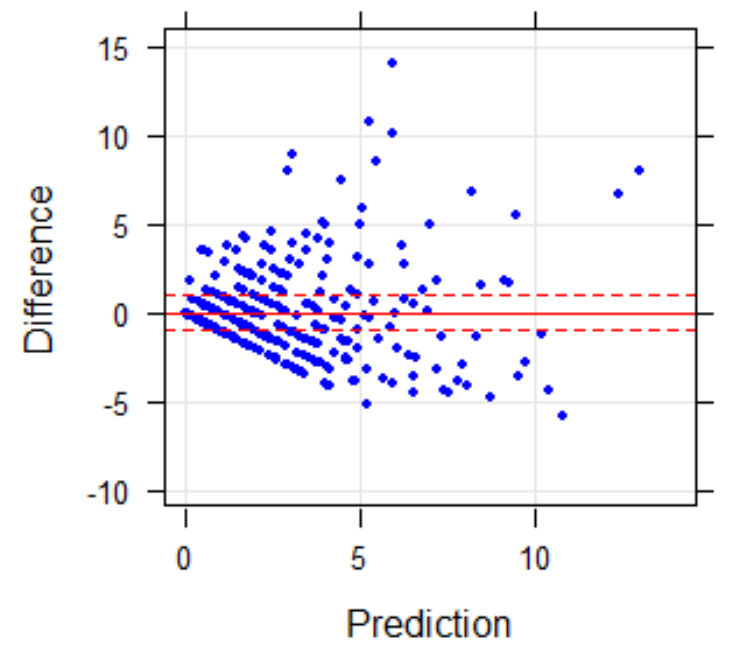

Rural Single Carriageways: Residuals

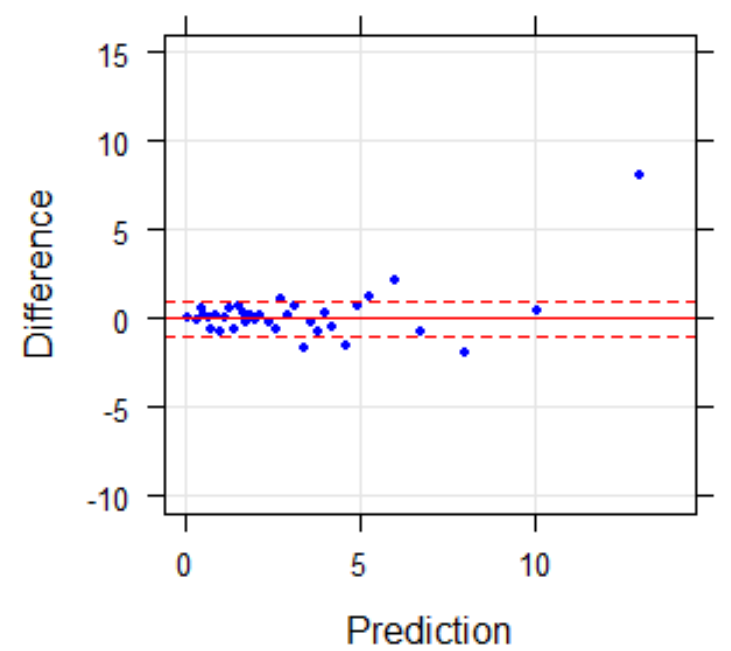

Figure 1. TRL model relating total accidents to an appropriate measure of flow with geometric design parameters included as explanatory variables. 